\title{
Eukaryotic Hibernating Ribosome Dimers are Maintained by a Kissing Loop Formed by Ribosomal RNA.
}

\author{
Wei Huang ${ }^{1}$, Dawid Krokowski², Maria Hatzoglou² and Derek Taylor ${ }^{1}$ \\ 1. Department of Pharmacology, Case Western Reserve University, Cleveland, OH. USA. \\ 2. Department of Genetics and Genome Sciences, Case Western Reserve University, Cleveland, $\mathrm{OH}$. \\ USA
}

Translational control of gene expression is a key regulator in the cellular response to environmental stimuli. To survive, cells react to stress conditions by globally and reversibly suppressing protein synthesis by placing ribosomes in an inactive hibernation state. In bacteria, the expression of stress-induced proteins promotes the formation of ribosome dimers to temporarily stall translation. For example, during the stationary growth phase, bacteria express a ribosome-modulation factor (RMF) or hibernation-promoting factor (HPF) that prevents translation of messenger RNA (mRNA) by coordinating the dimerization of two ribosomes $[1,2]$. Mechanistically, RMF/HPF blocks protein synthesis by occupying the decoding center in the aminoacyl-site of the small ribosomal subunit, whereas the dimerization event, which is governed by homodimerization of RMF or HPF, stabilizes the ribosomes in a protected state that prevents degradation of the translationally silent complex [3-5].

Hibernating ribosome dimers form in eukaryotes as well, and similarly in a manner that is enhanced by stress conditions [6]. Unlike the case of bacteria, however, a protein factor regulating hibernating dimeric ribosomes in eukaryotes has not been identified. Using a multidisciplinary approach, we show that ribosome dimers in nutrient deprived C6 glioma cells form through a novel and reversible interaction that is coordinated exclusively through eukaryotic-specific, ribosomal RNA (rRNA) expansion segments (ES). Our data reveal that this interaction is sequence specific and is coordinated by an intermolecular kissing hairpin that is formed by base-pairing between two ESs that reside within the large subunits of dimeric ribosomes. Our data provide molecular insights into a eukaryotic ribosome interaction that forms in response to stress and is regulated exclusively by RNA.

To understand the molecular mechanisms that contribute to translational control in eukaryotes, we exposed C6 glioma cells to nutrient deprivation conditions to promote dimerization of hibernating ribosomes. The dimer fraction was isolated by sucrose gradient sedimentation and the structure of the dimeric particles was solved using cryo-electron microscopy (cryo-EM) and single-particle reconstruction techniques. The structure identifies the intermolecular interaction, which localizes to the large ribosomal subunit, near the mRNA exit-site (Figure 1A). As the resolution of the dimer structure ( $8 \AA$ ) was hindered due to flexibility of the region connecting the two ribosomes, we used focused-classification to resolve a single ribosome within the dimer. Refinement of this structure to $3.3 \AA$ resolution provided superior detail to better assess the molecular interaction that coordinates dimer formation. In doing so we identified that the dimer interaction is orchestrated by the b-arm of ES31 (ES31b) of the large subunit rRNA.

ES31b forms a hairpin structure with an extended GC-rich loop at its apex. Based on this sequence, we predicted that separate ES31b loops could form intermolecular base-pairing interactions. To test our hypothesis, we in vitro transcribed the 30-nucleotide (nt) RNA exhibiting ES31b sequence, as well as a second construct that contained a series of base substitutions that were designed to disrupt the ability to base-pair with other hairpins (Figure 1B). Each construct was subjected to small angle x-ray scattering 
(SAXS) and the molecular envelope was determined (Figure 1C). These data revealed that the native RNA forms a complex that is almost twice as long as the mutant RNA (Figure 1B \& 1C). These results support a notion that the native ES31b sequence facilitates intermolecular dimerization events, most likely through base-pairing interactions, while the mutant RNA hairpin does not.

We next hypothesized that the individual RNA hairpins might compete for dimeric interactions with assembled ribosomes under stress conditions. C6 glioma cells were subjected to nutrient deprivation conditions to induce stress and to promote hibernating dimer ribosome formation. The ribosomes were then isolated on a sucrose gradient, which revealed the strong presence of dimeric ribosomes over their monomeric counterparts (Figure 1D, top panel). Similar experiments conducted in the presence of the 30nt ES31b RNA hairpin was sufficient to disrupt ribosome-ribosome interactions (Figure 1D, middle panel), while the mutant RNA did not affect ribosome dimer formation (Figure 1D, bottom panel). Together, these data support the role of the ES31b RNA sequence base-pairing with identical hairpins to govern ribosome dimer interactions in eukaryotic cells as part of the inherent stress response pathway.

References:

[1] Yoshida, H. et al, Genes Cells 14(2) (2009), p. 271.

[2] Ueta, M. et al, J Biochem 143(3) (2008), p. 425.

[3] Franken, L.E. et al, Nat Commun 8(1) (2017), p. 722.

[4] Kato, T. et al, Structure 18(6) (2010), p. 719.

[5] Matzov, D. et al, Nat Commun 8(1) (2017), p. 723.

[6] Krokowski, D. et al, Cell Cycle 10(16) (2011), p. 2691.
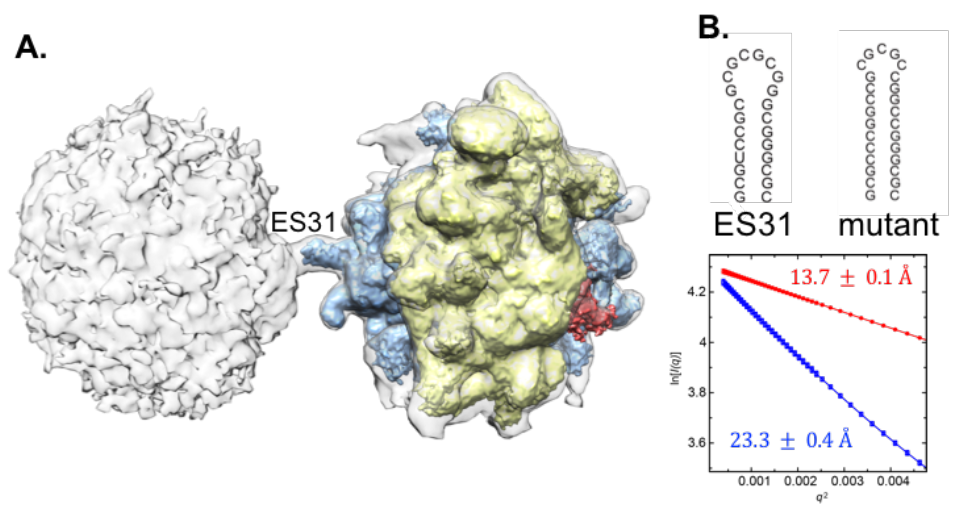

C.

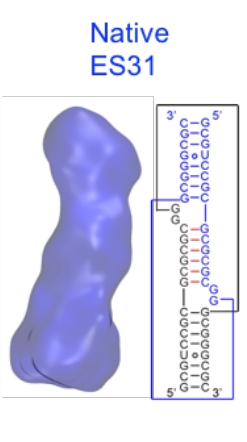

D.
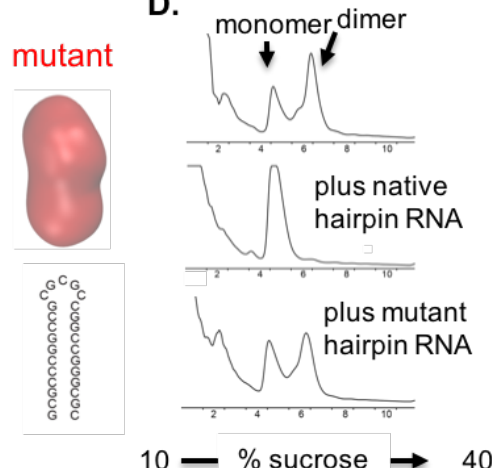

Figure 1. Structural and biophysical analysis of eukaryotic hibernating ribosome dimers. In contrast to bacterial ribosome dimers that are formed by a specific-protein that maintains a connection between two small ribosomal subunits, the structure here indicates that the intermolecular interaction is coordinated by a eukaryotic-specific ribosomal RNA expansion segment (ES31) that resides within the large subunit of the ribosomes $(\boldsymbol{A})$. SAXS analysis of a wild-type RNA hairpin with ES31b sequence or an RNA mutant reveals significant differences in biophysical properties of the two RNA constructs $(\boldsymbol{B})$. Molecular envelope calculations of the SAXS data support a role for the wild-type RNA to form dimers, through a hairpin kissing loop, while the RNA mutant exists as a monomer $(\boldsymbol{C})$. The individual RNA molecules were added to sucrose gradients of hibernating ribosome dimers. The RNA with native ES31b sequence disrupted dimer formation of stressed ribosomes, whereas the mutant RNA hairpin did not (D). 